\title{
Bone mesenchymal stem cell-derived exosomal microRNA-29b-3p prevents hypoxic-ischemic injury in rat brain by activating the PTEN-mediated Akt signaling pathway
}

Kun Hou', Guichen $\mathrm{Li}^{2}$, Jinchuan Zhao', Baofeng $\mathrm{Xu}^{1}$, Yang Zhang', Jinlu Y $\mathrm{u}^{1 *}$ and 'an $\mathrm{Xu}$ '

\section{Abstract}

Background: Mesenchymal stem cells (MSCs) are suspected to exert neuropro tive effects in brain injury, in part through the secretion of extracellular vesicles like exosomes containin active compounds. We now investigate the mechanism by which bone marrow MSCs (BMSCs)-derived exosomes has, Soring the small non-coding RNA miR29b-3p protect against hypoxic-ischemic brain injury in rats.

Methods: We established a rat model of middle cerebral rtery clusion (MCAO) and primary cortical neuron or brain microvascular endothelial cell (BMEC) models of oxys anc glucose deprivation (OGD). Exosomes were isolated from the culture medium of BMSCs. We treated the $\mathrm{AO}$ rats with BMSC-derived exosomes in vivo, and likewise the OGD-treated neurons and BMECs in features using TUNEL and CD31 immunohist chemica taining and in vitro Matrigel angiogenesis assays.

Results: The dual luciferase reporter gene assa chowed that miR-29b-3p targeted the protein phosphatase and tensin homolog (PTEN). miR-29b-3p was downres ated and PTEN was upregulated in the brain of MCAO rats and in OGD-treated cultured neurons. M AO rats and OGD-treated neurons showed promoted apoptosis and decreased angiogenesis, but overex ssion of miR-29b-3p or silencing of PTEN could reverse these alterations. Furthermore, miR-29b-3p could negatin, regulate PTEN and activate the Akt signaling pathway. BMSCs-derived exosomes also exerted protect vin a cts against apoptosis of OGD neurons and cell apoptosis in the brain samples from MCAO rats, where we also bbserved promotion of angiogenesis.

Conclusion: BMSC- rive exoscmal miR-29b-3p ameliorates ischemic brain injury by promoting angiogenesis and suppressing neurs val optosis, a finding which may be of great significance in the treatment of hypoxic-ischemic brain injury.

Keyword - Aypox, schemic brain injury, microRNA-29b-3p, Bone mesenchymal stem cells, Angiogenesis, Exosome, PTEN, kt signaling pathway

* Correspondence: jlyu@jlu.edu.cn; xu_xukan@126.com

'Department of Neurosurgery, The First Hospital of Jilin University, No. 1

Xinmin Avenue, Changchun 130021, Jilin, People's Republic of China

Full list of author information is available at the end of the article

(c) The Author(s). 2020 Open Access This article is distributed under the terms of the Creative Commons Attribution 4.0 International License (http://creativecommons.org/licenses/by/4.0/), which permits unrestricted use, distribution, and reproduction in any medium, provided you give appropriate credit to the original author(s) and the source, provide a link to the Creative Commons license, and indicate if changes were made. The Creative Commons Public Domain Dedication waiver (http://creativecommons.org/publicdomain/zero/1.0/) applies to the data made available in this article, unless otherwise stated. 


\section{Background}

Stroke is a common neurological disease that often leads to persistent disability, and is the third most common cause of death worldwide [1]. Acute ischemic stroke, which accounts for more than $80 \%$ of all stroke cases, may result in permanent brain damage when restoration of circulation is delayed [2]. Most cases of cerebral stroke result from transient or permanent occlusion of a cerebral blood vessel, which eventually proceeds to brain infarction [3]. Nowadays, the first-line treatment for ischemic stroke is thrombolysis with intravenous tissue plasminogen activator. However, the restoration of blood flow may under certain circumstances exacerbate oxidative stress and inflammatory responses $[4,5]$. Therefore, there is an urgent need for innovative stroke treatments.

It is well-recognized that the route to hypoxic-ischemic brain injury is a complicated process involving various biological pathways both in the early and late stages [6]. Among the involved pathways, angiogenesis and neurogenesis are now principal targets in the treatment of stroke [7]. Transplantation with mesenchymal stem cells (MSCs) is reportedly a candidate target in the treatment of neonatal hypoxicischemic brain injury, and is increasingly drawing the attention of stroke researchers [8]. The exosomes derived from MSCs mediate the therapeutic actions of MSCs $[9,10]$. In recent years, growing evidence has suggested that microR NAs (miRNAs) play critical roles in ischemic stroke [11] mental therapeutic approaches based on miRNAs 1tave developed to help post-stroke neurological a very an, ameliorate ischemic brain injury $[12,13]$. miR-29bthe members of the miR-29b family to te identified, es hibits therapeutic potential for treating card metabolic disorders [14]. Furthermore, the expression of $29 \mathrm{~b} / 3 \mathrm{p}$ was reduced in patients with intestinal hemia-reperfusion injury [15]. The gene for phosphatase an t t homolog (PETN), which is located on hum an romome 19, encodes a dual phosphatase enzyme th de nothorylates both protein lipid substrates [13. Alsu TTEN is a negative regulator of the phosphatid sitol 3-1, nase/protein kinase B (PI3K/ Akt) signaling path $\left.{ }^{17}\right]$, which is implicated in hypoxicischemic injury [18]. F,owever, it has been unclear whether bone ma w nes nchymal stem cells (BMSCs)-derived exosor and $R \cdot 29 \mathrm{~b}-3 \mathrm{p}$ moderate the severity of ischemic rain aiury and whether their regulation is dependent on P. Vor we Akt signaling pathway. Given that PTEN regulates glogenesis in tumors and healthy tissues, it is notable that angiogenesis of brain microvascular endothelial cells (BMECs) is necessary for the functional restoration of brain injury, such as ischemic stroke [19]. Therefore, we conducted extensive experiments to test the hypothesis that BMSCderived exosomal miR-29b-3p participates in the neuronal apoptosis and angiogenesis of BMECs in the context of hypoxic-ischemic brain injury, which might be associated with PTEN and the Akt signaling pathway.

\section{Materials and methods}

\section{Ethics statement}

The present study was approved by the Ethics Committee of The First Hospital of Jilin University. All procedures involving animals were conducted in line with the regulations of the institutional animal care and us ${ }^{\text {c com- }}$ mittee (ethics number: 2018-415).

Rat middle cerebral artery occlusion mode' establish and neurobehavioral evaluation

A permanent model of middle cereloral ar occlusion (MCAO) was established accordir $;$ to the pr viously reported Longa method [20, 21], u g 32 hale SpragueDawley (SD) rats (aged 6-8 eks, al weight $200 \pm$ 15 g, Shanghai Sippr B' Labor ry Animals Co., Ltd., Shanghai, China) as pro usly reported [22]. The rats used in the experiment housed under specificpathogen-free S SPr conditions in a 12-h light/dark cycle, with free food and water. The MCAO procedure was $a_{p}$ ied only when the rats reached a body weig $\{250 \leq 12 \mathrm{~g}$. Then, a thread plug was prepared with a 27 - nm-long 3-0 nylon thread. The tip was heated on $\mathrm{n}$ alcohol burner to form a plug of hemiSp. cal bulb shape with a diameter of $0.33 \mathrm{~mm}$, which vas s leared with silicone grease. Rats were anesthetized h $2 \%$ pentobarbital sodium at a dose of $30 \mathrm{mg} / \mathrm{kg}$ (i.p.) before they were fixed on the operating table in the supine position. The right common carotid artery (CCA), internal carotid artery (ICA), and external carotid artery (ECA) were separated. The ECA and CCA were ligated. After clamping the distal end of the ICA, a cut was quickly made at the bifurcation of ECA and ICA. The nylon thread (a mark was made $2 \mathrm{~cm}$ from the heated bulb end) was inserted from the incision into the ICA. The nylon thread and the ICA were ligated slightly at the incision. Then, the arterial clamp that clamped the ICA was loosened. The nylon thread was continued to be inserted until a slight resistance was felt (the insertion depth was about $18.5 \pm 0.5 \mathrm{~mm}$ ), and it was pulled out slightly. Thus, cerebral ischemia was developed due to middle cerebral artery occlusion. The neurological state of the MCAO rats was assessed within 1-2 h upon they awakened from anesthesia (about 30 min after operation). According to Bederson's behavioral scoring method [23], the neurological function of the rats was evaluated by a double-blind scoring method, as follows: (1) no central nervous system injury symptoms (0 point); (2) unable to fully extend the contralateral front claw (1 point); (3) circling toward the side opposite the surgery (2 points); (4) falling to the side away from the surgery (3 points); (5) unable to walk spontaneously or failure to regain consciousness (4 points). A total of 32 rats were used for modeling, and the rats with scores in the range of 1-3 were regarded as successfully modeled MCAO 
rats $(28 / 32 ; 87.5 \%)$, and the others were excluded from the subsequent experiments. The successfully modeled rats were randomly assigned into four groups $(n=7$ in each group). Rats assigned to the sham group $(n=7)$ were subjected to the procedures of isolating carotid arteries, but without the insertion of a nylon thread.

Intracerebroventricular stereotactic injection of MSCsderived exosome (MSCs-Exo) or MSCs-Exo-miR-29b-3p agomir at lateral ventricle was carried out $2 \mathrm{~h}$ after the MCAO model was first developed, i.e., immediately after behavioral assessment. Rats were anesthetized as above mentioned, whereupon their heads were fixed on the stereotaxic instrument. Then, hair on the scalp was removed, and the skin was disinfected with iodophor and $75 \%$ alcohol. An incision measuring $1.5 \mathrm{~cm}$ was cut along the midline of the head to expose the skull around the Bregma. According to the stereotactic atlas of the rat, a trocar was inserted into the lateral ventricle (0.8 $\mathrm{mm}$ posterior to the Bregma, $1.5 \mathrm{~mm}$ next to the midline, $4.0 \mathrm{~mm}$ below the outer surface of the skull) [21]. The trocar was reserved for further use. The rats were placed in a warmed box with free access to food and water. One week later, the rats were administered by trocar. An indwelling needle was reserved for future use. The MSCs-Exo or MSCs-Exo-miR-29b-3p agomir was injected one time every day for 3 days at a doce of $100 \mu \mathrm{g} / \mathrm{kg} /$ day [24]. After another $72 \mathrm{~h}$, the rats wh el thanized, and the brains were collected and dirated. portions used for 2,3,5-triphenyl tetrazol a chloria (TTC) staining, immunohistochemistry, ns weli RNA and protein isolation.

\section{Isolation of primary cortical neurons, b Cs a d BMSCs}

Primary cortical neurons were lated from the cerebral cortices under SPF conditions of (et) 1 aged 16-18 days using a modification of /a 's method [25]. Then the isolated cortices were ch nto mall pieces, mixed with Dulbecco's modifief Eas "s medium (DMEM), and transferred to a vrile cur are bottle, followed by trituration with a pasteu d tube 30 times. Next, the suspension was subjected to centrifugation at $1000 \mathrm{~g}$ for $5 \mathrm{~min}$. The isor. dells were cultured at $37{ }^{\circ} \mathrm{C}$ with $5 \% \mathrm{CO}_{2}$ at a der 10 of cells/well on culture plates which had ren oated with $50 \mu \mathrm{g} / \mathrm{mL}$ poly- $L$-lysine prior to use. A. Incubation for $4 \mathrm{~h}$, the medium was renewed with neur specific culture medium. Finally, the growth of the cells was observed under a microscope.

For BMEC isolation, the SD rats (aged 6-8 weeks, weighing $200-250 \mathrm{~g}$ ) were euthanized by intraperitoneal injection of an overdose of pentobarbital sodium $(6 \%$, $90 \mathrm{mg} / \mathrm{kg}$ ). The cerebral cortex was dissected and immersed in cold D-Hanks and DMEM. The tissues were cut into pieces and water-bathed with $0.1 \%$ type II collagenase for $1.5 \mathrm{~h}$. After centrifugation at $1000 \mathrm{~g}$ at room temperature for $8 \mathrm{~min}$, the tissue blocks were suspended in $20 \%$ bovine serum albumin (BSA) and centrifuged at $1000 \mathrm{~g}$ at $4{ }^{\circ} \mathrm{C}$ for $20 \mathrm{~min}$. Then the tissue blocks were water-bathed again with $0.1 \%$ collagenase/ dispase at $37{ }^{\circ} \mathrm{C}$ for $1 \mathrm{~h}$. After a recentrifugation at $1000 \mathrm{~g}$ at room temperature for $8 \mathrm{~min}$, the tissue locks were resuspended in $2 \mathrm{~mL}$ DMEM and cor ed with $12 \mathrm{~mL} \mathrm{50 \%} \mathrm{Percoll.} \mathrm{The} \mathrm{microvascular} \mathrm{segmen} \mathrm{were}$ removed by centrifugation twice with DMEM at $1 / \mathrm{g}$ at room temperature for $5 \mathrm{~min}$. The tained pellets were suspended in DMEM complete cul s nedium, seeded in culture dishes pre-co ed with g-atin substrate, and cultured in an iricul or with $5 \% \mathrm{CO}_{2}$ at $37^{\circ} \mathrm{C}$. After $24 \mathrm{~h}$, the medil was anged with fresh medium supplemented with $1 \mathrm{r} / \mathrm{mL}$ brain fibroblast growth factor. Every her day, the medium was renewed. Cells at passage $t_{1}$ ewere used for animal experiments and $x \mathrm{~s}$ he isolation.

Adult male $r$ jed 6-8 weeks, weighing 200$250 \mathrm{~g}$ ) were eutha ed as above and bone marrow was obtained insing the fractured femur of rats with DMEM (Hycion, 2, Marlborough, MA, USA) containing $10 \%$ fetal bovine serum (FBS). The marrow cells were cur ed in DMEM containing 10\% FBS plus $1 \%$ enic in-streptomycin (P1400, Solarbio, Beijing, China) a detached with $0.25 \%$ trypsin plus ethylene diamine tetraacetic acid (concentration). The culture media were ultra-centrifugated at $120,000 \mathrm{~g}$ at $4{ }^{\circ} \mathrm{C}$ for $2 \mathrm{~h}$ to isolate the serum exosomes before use.

\section{Neuron oxygen-glucose deprivation model}

We performed the oxygen-glucose deprivation (OGD) treatment in rat primary cortical neurons and BMECs as previously reported [22]. For OGD treatment, the culture medium was renewed with ischemic-mimetic D-Hanks containing $140 \mathrm{mM} \mathrm{NaCl}, 3.5 \mathrm{mM} \mathrm{KCl}, 0.43 \mathrm{mM}$ $\mathrm{KH}_{2} \mathrm{PO}_{4}, 1.25 \mathrm{mM} \mathrm{MgSO}, 1.7 \mathrm{mM} \mathrm{CaCl}, 5 \mathrm{mM}$ $\mathrm{NaHCO}_{3}$, and $20 \mathrm{mM} \mathrm{N}$-2-hydroxyethylpiperazine- $\mathrm{N}^{\prime}$-2ethanesulfonic acid $(\mathrm{pH}=7.2-7.4)$. Then cells were cultured in a hypoxic incubator supplemented with $95 \% \mathrm{~N}_{2} /$ $5 \% \mathrm{CO}_{2}$ for $6 \mathrm{~h}$ at $37^{\circ} \mathrm{C}$ (Billups-Rothenberg, San Diego, CA, USA) and collected immediately thereafter $[26,27]$.

\section{Cell transfection}

When the BMSCs had reached $80-90 \%$ confluence, the cells were transfected according to the instructions for Lipofectamine 2000 (11668-019, Invitrogen, New York, CA, USA). Group 1: negative control (NC) mimic group (BMSCs treated with NC mimic), miR-29b-3p mimic group (BMSCs treated with miR-29b-3p mimic), NC inhibitor group (BMSCs treated with NC inhibitor), and miR-29b-3p inhibitor group (BMSCs treated with miR29b-3p inhibitor). Group 2: OGD + BMSCs + miR-29b$3 p$ mimic NC group (OGD cells co-cultured with 
exosome of BMSCs treated with NC mimic), OGD + BMSCs + miR-29b-3p mimic group (OGD cells cocultured with exosome of BMSCs treated with miR-29b3p mimic), OGD + BMSCs + miR-29b-3p inhibitor NC group (OGD cells co-cultured with exosome of BMSCs treated with miR-29b-3p inhibitor $\mathrm{NC}$ ), and OGD + BMSCs + miR-29b-3p inhibitor group (OGD cells transfected with exosome of BMSCs treated with miR-29b-3p inhibitor). The mimic and inhibitor of miR-29b-3p were purchased from Ribobio Company (https://www.ribobio. $\mathrm{com} /$ ) and transfected at a concentration of $100 \mathrm{nM}$.

\section{Lentivirus construction and infection}

The lentiviruses harboring miR-29b-3p overexpression and shRNA targeting PTEN were purchased from Genechem (Shanghai, China). Neurons or endothelial cells were infected with lentivirus. The subsequent experiments were carried out $24 \mathrm{~h}$ after infection.

\section{Extraction, identification, and labeling of exosomes}

BMSCs-derived exosomes were extracted as previously reported [28], and the morphology and size distribution of exosomes were observed by transmission electron microscopy and dynamic light scattering (DLS) analysis. The exosome markers were assessed by Western blot analysis. PKH-67 labelling was performed followin the PKH-67 kit instructions (MINI61-KT, Sigma-Aldri

Louis, MO, USA). In brief, the exosome wa incuba with PKH67-Diluent $\mathrm{C}$ for $5 \mathrm{~min}$, and the $\mathrm{S}$ ing was terminated with $0.5 \%$ BSA. Then the exolome w nurified with the exosome extraction ki and the PKH-67labeled exosome was obtained. Ne ons arld BMECs were incubated with PKH-67-labeled for $24 \mathrm{~h}$. After being fixed in $4 \%$ pa maldehyde at room temperature for $30 \mathrm{~min}$, cells we re sta, ned by 4',6-diamidino-2-phenylindole 25.c1 API; 6308ES11, Yeasen Biotechnology Co., Ltd., by observation u der an . erted fluorescence microscopy (DMi8, Leica, Wu 'ar, Ger/nany) and photographed.

TTC st

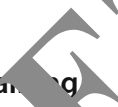

The hrain ue were obtained from rats $(n=7$ in each roup and gat onto 1-mm-thick sections. TTC solution (2 rom, Solarbio, Beijing, China) was added to the brain tissu ctions for a $10-15$-min incubation at $37^{\circ} \mathrm{C}$, and the sections were fixed using $4 \%$ paraformaldehyde, observed microscopically, and analyzed with the ImageJ software.

\section{Terminal deoxyribonucleotidyl transferase-mediated biotin-16-dUTP nick-end labelling assay}

The apoptosis of tissues was evaluated following the cell apoptosis detection kit instructions (TUN11684817, Roche, Mannheim, Germany). The tissue sections were dewaxed, rehydrated, and pretreated with protease- $\mathrm{K}$ for $30 \mathrm{~min}$, followed by terminal deoxynucleotidyl transferase dUTP nick end labeling (TUNEL) staining for apoptosis detection at $37{ }^{\circ} \mathrm{C}$ for $1 \mathrm{~h}$. Converter-pod was added to the sections followed by incubation at $37^{\circ} \mathrm{C}$ for $30 \mathrm{~min}$, and washing with phosphate-buffered saline (PBS) incubation with diaminobenzidine for $10 \mathrm{~min}$. The sections were then counter-stained with hematoxylin, rinso tehydrated, and sealed. With a drop of PBS or/glycerin $\mathrm{p}$ d in the field of vision, apoptotic cells (200 00 cell ) were observed and photographed by optipal micr opy (IX53, Olympus Optical Co., Ltd., Tokyo Japan). We) judged the morphological characteristics of $t$ apoptotic cells; unstained cells were small witn tach mbrane but with the foaming phenomen n, who apoptotic bodies first appear at a late stage $\mathrm{wh}_{\mathrm{L}}$ the adherent cells being crumpled, rounded, and felling The stained cells exhibit chromatin condensic on, with marginalization and lysed nuclear mentb $\quad$ with chromatin divided into masses/apoptotic ties. Five sections of each rat brain sample we ndonily selected for assessment.

The pre-ireatn, nt of the cultured cells was conducted as follows: the vides were coated with a thin layer of polylySin ir-dried, rinsed in deionized water, and stored at $4{ }^{\circ} \mathrm{C}$. tpop sis was induced by the appropriate method, and vas a negative control (NC) group without induction of cell apoptosis. About $1 \times 10^{6}$ cells were collected by centrifugation, washed in PBS, resuspended, transferred to the coated slides, and air-dried. The slides were then fixed in 4\% paraformaldehyde for $25 \mathrm{~min}$ and treated with $0.2 \%$ Triton X-100 for $5 \mathrm{~min}$, with subsequent procedures the same as those for paraffin-embedded section.

\section{RNA isolation and reverse transcription quantitative polymerase chain reaction}

Total RNA from cells and tissues, and exosome-derived RNA were extracted using TRIZOL (15596-018, Beijing Solabio Life Sciences Co., Ltd., Beijing, China). The primers used in this study were synthesized by Takara Company (Dalian, Liaoning, China) (Table 1). Using GAPDH and U6 as internal reference primers, the relative mRNA expression of target genes was calculated by the $2^{-\triangle \Delta \mathrm{Ct}}$ method [29].

\section{Western blot analysis}

Total proteins were extracted from tissues, cells, or exosomes by radioimmunoprecipitation assay lysis buffer (R0010, Beijing Solabio Life Sciences Co., Ltd., Beijing, China). The protein concentration was assessed through a bicinchoninic acid kit (20201ES76, Yeasen Biotechnology Co., Ltd., Shanghai, China). After being separated by polyacrylamide gel electrophoresis, the protein was transferred to a polyvinylidene fluoride membrane (Merck Millipore, Billerica, MA, USA) which was blocked in 5\% BSA for one $\mathrm{h}$ at room temperature and then incubated with primary rabbit 
Table 1 Primer sequence for RT-qPCR

\begin{tabular}{|c|c|c|}
\hline miRNA/mRNA & Sequence $\left(5^{\prime}-3^{\prime}\right)$ & \\
\hline miR-29b-3p & F: TTCCTATGCATATACTTCT & R: CGTATCCAGTGCGAATA \\
\hline PTEN & F: ATCTTGTGCTCACCCTGACA & R: ACCTCTCGGGAGTACACACT \\
\hline GAPDH & F: CTGACATGCCGCCTGGAGA & R: ATGTAGGCCATGAGGTCCAC \\
\hline U6 & F: ATGACGTCTGCCTTGGAGAAC & R: TCAGTGTGCTA \\
\hline $\mathrm{BCl}-2$ & F: AGCCCTGTGCCACCTGTGGT & R: ACTGGACATC \\
\hline Bax & F: AACAACATGGAGCTGCAGAGG & R: GAAGT \\
\hline Caspase-3 & F: TACCCTGAAATGGGCTTGTGT & $\mathrm{R}: \mathrm{GTT}$ \\
\hline
\end{tabular}
$B a x \mathrm{Bcl}-2$ associated $\mathrm{X}, R$ reverse, $F$ forward

antibodies against TSG101 (ab30871, 1:1000), CD80 (ab109201, 1:1000), vascular endothelial growth factor A (VEGFA; ab46154, 1:1000), vascular endothelial growth factor receptor 2 (VEGFR2; ab11939, 1:1000), caspase 3 (ab13847, 1:1000), B cell leukemia 2 (Bcl-2; ab196495, 1: 1000), Bcl-2 associated X (Bax; ab32503, 1:2000), Akt (ab8805, 1:1000) and p-Akt (ab38449, 1:1000), rat antibodies against CD63 (ab108950, 1:1000), and glyceraldehyde-3phosphate dehydrogenase (GAPDH; ab8245, 1:5000). The membrane was then incubated with horseradish peroxidaselabeled goat anti-rabbit (ab205718, 1:10,000) or goat antimouse (ab6789, 1:5000) secondary antibody at room temperature for $1 \mathrm{~h}$. The above antibodies were purchased from Abcam (Cambridge, UK). Bands were develor an 1 band intensity was quantified using Image 1.48 a soft (National Institutes of Health, Bethesda, MD, SA), wit, GAPDH used as an internal reference standnra.

\section{Dual luciferase reporter gene assay}

Reporter gene vectors of wild-twe ana _...ung site mutated (pGL3-PTEN-661 Wt/pCL pGL3-PTEN-1703 Wt/pCI 3-PTE $/ 1753$ Mut) were constructed and transfecte wi miR- $9 \mathrm{~b}-3 \mathrm{p}$ mimic and pRLTK (internal refernnce expressing luciferase of Renilla) in HEK? $3 \mathrm{~T}$ cells merican Type Culture Collection, Manassa, h USA). Twenty-four hours later, cells were lysed according $\delta$ the instructions of TransDetect Double cil rase Reporter Assay Kit (FR201-01, TransGen Biote Beii,ng, China) and the supernatant was collectec o dety the activities of firefly (FL) and renilla (RL) $1 . f_{e}$ the Dual-Luciferase Reporter Assay System (E1 Promega, Madison, Wis., USA). The ratio of FL/RL was ured as the relative luciferase activity.

\section{Tube formation assay}

Cells were starved with serum-free medium for $24 \mathrm{~h}$ in cell suspension $\left(1 \times 10^{5}\right.$ cells $\left./ \mathrm{mL}\right)$ and cultured in 24well plate coated with Matrigel (354234, Shanghai Shanran Biotechnology Co., Ltd., Shanghai, China) for $6 \mathrm{~h}$. Capillary-like tube structures were identified under a
Leica inverted phase contras icro pe $(\times 100)$. Tube length and branch poin were c. ulated at five random fields using Image- $\operatorname{Pr}, 6.0$ sotcware.

\section{Immunohistoch mis}

The expression ab24590, 1:100, Abcam, Cambridge, UK) and ए N (ab170941, 1:100, Abcam, Cambridge, Ul detected by routine immunohistochemical staining. The 0,031 expression was observed under a microscope (LX53, Olympus Optical Co., Ltd., Tokyo, Tap and microvessel density (MVD) was calculated by the $\mathrm{m}$ thod of Weidner [30]. CD31 was mainly expressed h. he cytoplasm/membrane of endothelial cells, presenting as Drown stain under the microscope. Five sections of each rat brain tissue were randomly selected for observation.

\section{Statistical analysis}

SPSS 21.0 statistical software (IBM Corp. Armonk, NY, USA) was used for data analysis. All data were tested for normal distribution and homogeneity of variance using Levene's test. The data conforming to the normal distribution were presented as mean \pm standard deviation. Unpaired two-tailed $t$ test was used for comparisons between two groups, and one-way analysis of variance (ANOVA) analysis was used for comparisons among multiple groups, followed by Tukey's post-hoc tests. The difference was statistically significant when $p<0.05$.

\section{Results}

Expression of miR-29b-3p is significantly decreased in the cerebral cortex of MCAO rats

A study has shown that miR-29b-3p is poorly expressed in intestinal ischemic injury models and reduces intestinal ischemic injury $[15,31]$. However, the role of miR-29b-3p has remained uncertain in cerebral ischemic injury. We established the rat model with MCAO to investigate the molecular mechanism of miR-29b-3p in the ischemic brain injury. After $72 \mathrm{~h}$, TTC staining showed that the brain of MCAO rats exhibited significant cerebral infarction compared with control sham rats $(p<0.05)$, suggesting that the ischemic brain injury model was successfully established (Fig. 1a, b). 


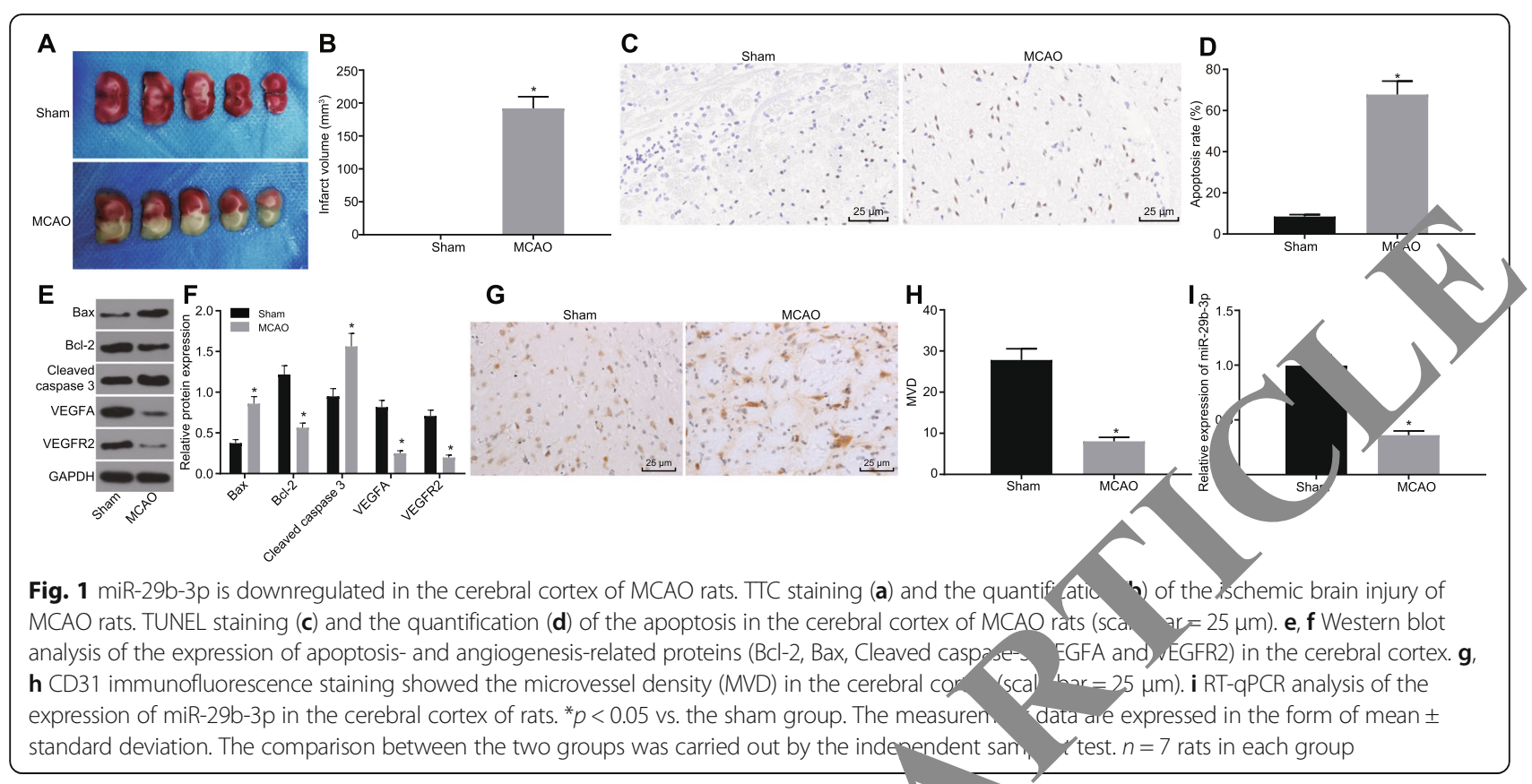

TUNEL staining was performed to detect the apoptosis in cerebral cortex. As shown in Fig. 1c, d, the apoptotic rate in the cortex of rats with MCAO was significantly increased $(p<0.05)$. Then we examined the apoptosis and angiogenesis-related proteins by Western blot anal, ss shown in Fig. 1e, f, the level of anti-apoptotic protein b ? was significantly inhibited in MCAO rats, y the pro apoptotic protein Bax was significantly increasea. esides, the level of cleaved caspase- 3 was also elevated, ino, cating that the apoptosis process was activ ed in the brain of $\mathrm{MCAO}$ rats. As for the angiogenesis-pro tino proteins, we found that the levels of VEGFA a ITEGFR2 were decreased significantly (Fig. 1e, f, $p<0.05$ ). unohistochemistry staining revealed that th $D$ incicated by CD31 staining was remarkably decre d $\mathrm{MCAO}$ rats (Fig. $1 \mathrm{~g}, \mathrm{~h}, p<$ 0.05). In addition, express of miR-29b-3p was significantly downregulated he infaro d brain tissue of MCAO rats versus that in the sh, operated rats (Fig. 1i, $p<0.05$ ). These findings aggest that $\mathrm{m}_{\Delta} \mathrm{R}-29 \mathrm{~b}-3 \mathrm{p}$ was decreased significantly in the $\mathrm{D}$ ape sis an tecreased angiogenesis.

$\mathrm{B}_{1}$ s reurce the neuron apoptosis and inhibit angiogenesis of Bly exposed to OGD by releasing exosomes

Since exosomes derived from MSCs have been reported to protect against various kinds of disorders [32, 33], we sought to explore whether BMSC-derived exosomes (MSCs-Exos) have beneficial influences on OGDinduced injury to neurons and BMECs. OGD treatment was initially conducted in primary cultured cortical neurons and BMECs. Then these cells were co-cultured with BMSCs to measure relevant indexes. First, we observed that the apentotic cell number was increased in OGDstr d cortical neurons (Fig. 2a, b). Besides, the expresion Bax was enhanced, while the expression of Bcl-2 Gecreased, whereas the activity of caspase-3 was activated in OGD cortical neurons (Fig. 2c, d). In addition, the levels of VEGFA and VEGFR2 were remarkably decreased (Fig. 2c, d), accompanied by inhibited tube formation of BMECs (Fig. 2e, f). Meanwhile, miR-29b-3p expression was significantly reduced in OGD cortical neurons (Fig. 2g). When co-cultured with BMSCs, the number of apoptotic cells was decreased in OGD cortical neurons, along with diminished expression of Bax and caspase- 3 and promoted expression of Bcl-2. In addition, the tube formation of BMECs was significantly restored by the treatment, as evidenced by upregulated VEGFA and VEGFR2 expression. While the addition of GW4869 (an inhibitor of exosome secretion) led to no significant difference relative to cells treated with OGD (Fig. 2a-f). Finally, the expression of miR-29b-3p in OGD cortical neurons and BMECs co-cultured with BMSCs was significantly upregulated (Fig. 2g). On the other hand, GW4869 affected the delivery of exosomes, leading miR-29b-3p expression reduced to the same level as cells without co-culture. Therefore, BMSCs could reduce the neuron apoptosis and reverse the suppression in angiogenesis of BMECs induced by OGD by releasing exosomes.

\section{BMSCs transfer miR-29b-3p to neurons and BMECs through exosomes}

Exosomes were extracted from culture medium of BMSCs at passage four, and the morphology was observed under 


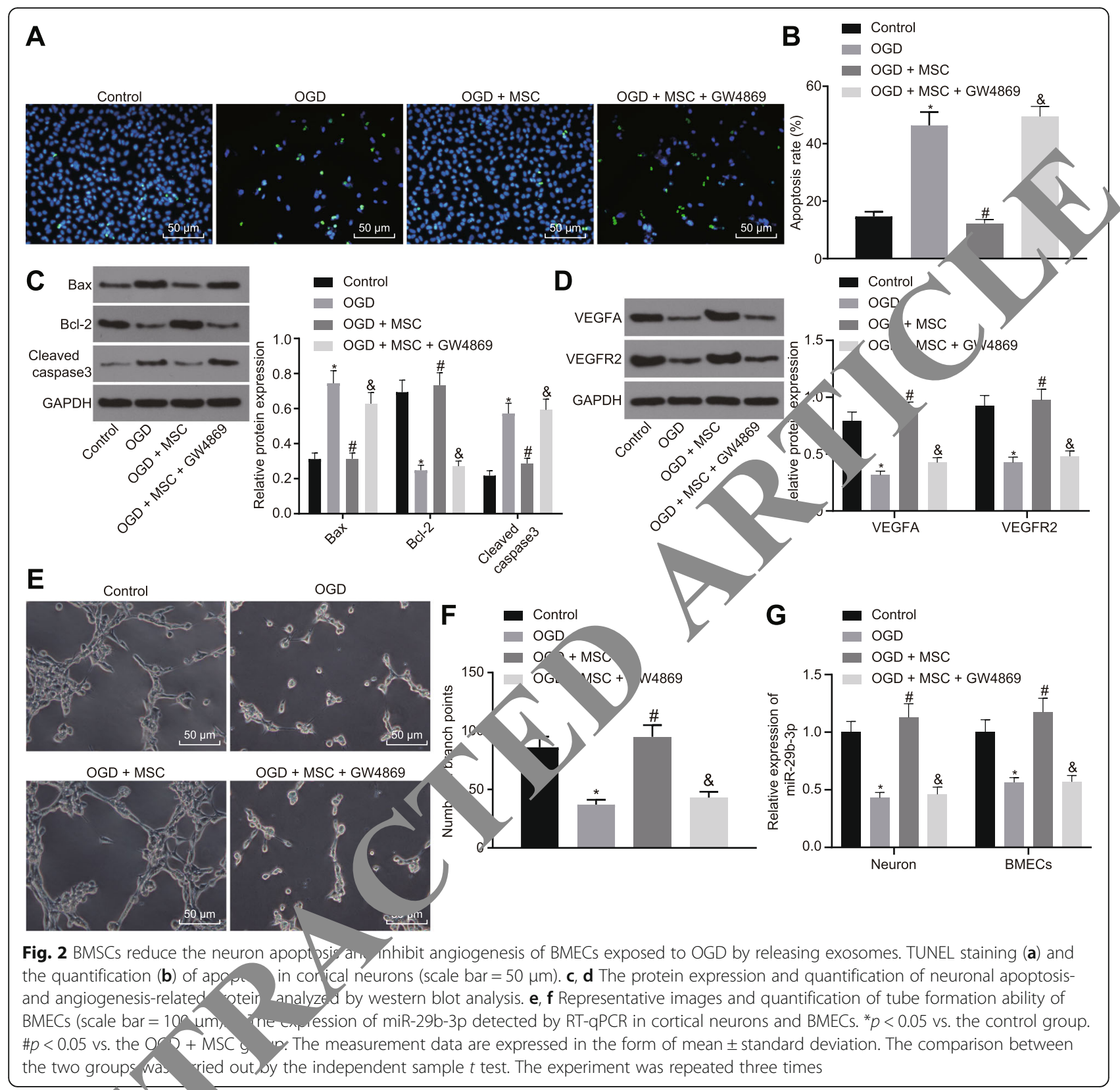

a transm is ele ctron microscopy (Fig. 3a). The size distrib in os romes was confirmed by electron microsэpy and dynamic light scattering (Fig. 3b), and the

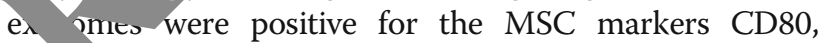
CD6. and TSG101, as verified by Western blot analysis (Fig. 3c). Thus, the exosomes were isolated successfully. Then, the BMSCs-derived exosomes were labeled with PKH-67 and added to the culture medium of cortical neurons or BMECs in the Transwell system. Four hours later, we observed a significant number of exosomes in neurons and BMECs (Fig. 3d). After $24 \mathrm{~h}$, the expression of BMSC markers CD29, CD90, and CD105 was significantly increased in neurons and BMECs treated with BMSCs- derived exosomes. In addition, compared with untreated neurons and BMECs, the expression of miR-29b-3p was also significantly elevated in neurons and BMECs treated with BMSCs-derived exosomes (Fig. 3e, f). As shown in Fig. 3g, the reverse transcription quantitative polymerase chain reaction (RT-qPCR) results showed that miR-29b$3 p$ expression was significantly upregulated in exosomes from BMSCs transfected with miR-29b-3p mimic and downregulated in exosomes from BMSCs transfected with miR-29b-3p inhibitor $(p<0.05)$. Similarly, after co-culture of BMSC-derived exosomes with neurons or BMECs, miR-29b-3p was significantly upregulated in neurons and BMECs co-cultured with exosomes from BMSCs 


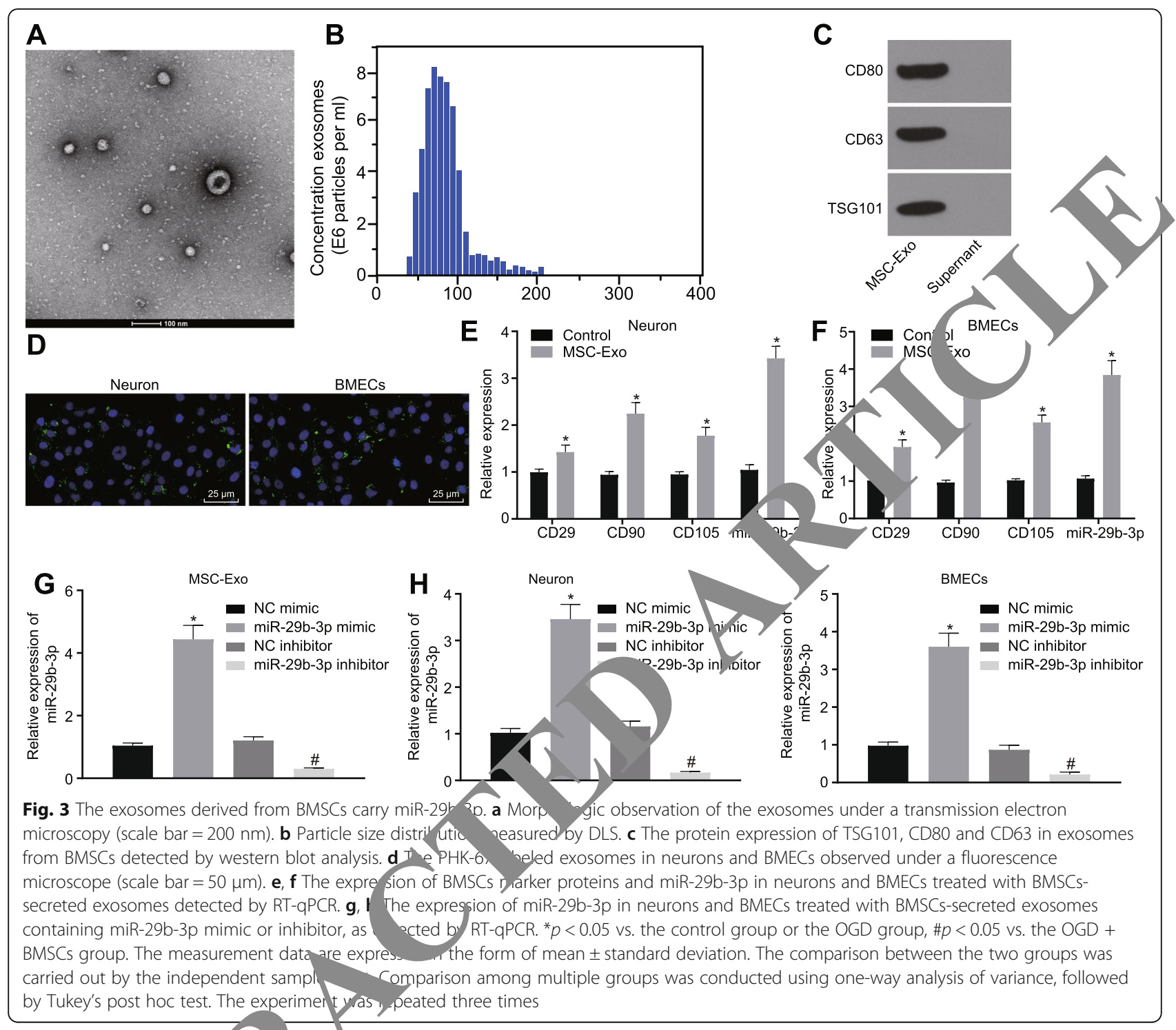

overexpressing miN-29b but downregulated after coculture with ones fom BMSCs transfected with miR-29b-3p inhiblu (Fig. 3h). Thus, the BMSCs could transfer exosome-shucted miR-29b-3p into neurons or BMECs.

\section{MSC -derived exosomal miR-29b-3p attenuates neuronal} a) tosis and promotes BMEC angiogenesis

To is Atify whether BMSCs-derived exosomal miR-29b$3 \mathrm{p}$ is related to regulation of neuronal apoptosis and BMEC angiogenesis, neurons and BMECs were treated with BMSCs-derived exosomes expressing $\mathrm{NC}$ mimic, miR-29b-3p mimic, NC inhibitor, or miR-29b-3p inhibitor, and then collected for further analysis. The TUNEL staining showed that the apoptosis of the neurons treated with MSCs-Exo miR-29b-3p mimic was lower than that of neurons treated with MSCs-Exo NC mimic, and that apoptosis was significantly increased in neurons treated with MSCs-Exo miR-29b-3p inhibitor compared with those treated with MSCs-Exo NC inhibitor (Fig. 4a, b). Furthermore, Western blot analysis confirmed that the expression of Bcl-2, VEGFA, and VEGFR2 in the cortical neurons was upregulated by MSCs-Exo miR-29b-3p mimic, and that the expression of Bax and cleaved caspase- 3 was inhibited. By contrast, co-culture with MSCs-Exosomes containing miR-29b-3p inhibitor contributed to diminished Bcl-2, VEGFA and VEGFR2, while promoting Bax and cleaved caspase-3 expression in cortical neurons (Fig. 4c, d). Tube formation assay revealed that the number of branch points was significantly increased following treatment of MSCsExo miR-29b-3p mimic, while the number of branch points after MSCs-Exo miR-29b-3p inhibitor was significantly reduced (Fig. 4e). Therefore, the exosomes released from BMSCs overexpression miR-29b-3p was able to ameliorate 


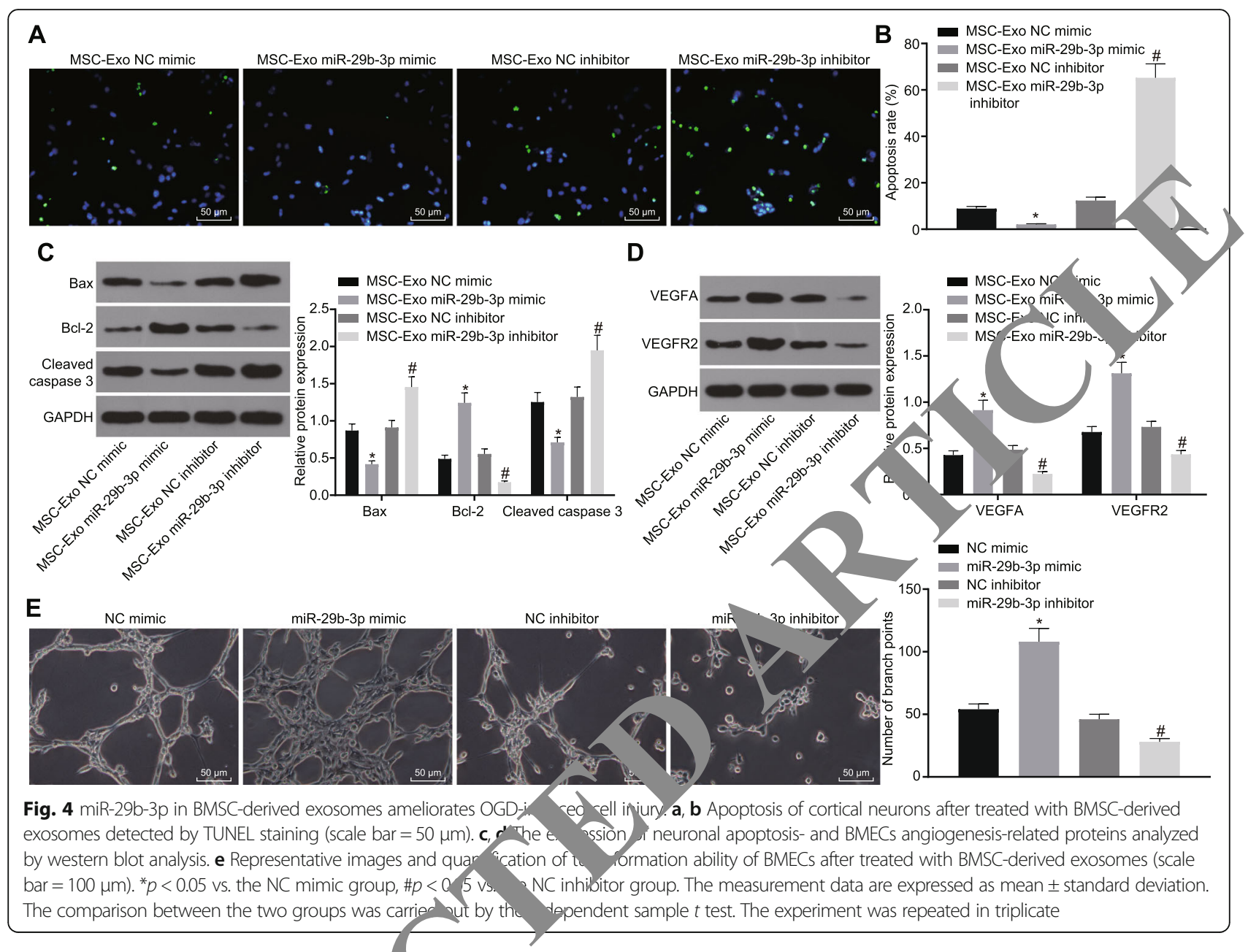

the neuron apoptosis and reverse su ssio in BMEC angiogenesis caused by OGD tre: ont.

miR-29b-3p targets $P$ to Ancrease neuron injury induced by OGD

The potential/ ding sis between miR-29b-3p and PTEN gene were edicted using TargetScan (http:// www.tar etscan.org), showing that miR-29b-3p could bind to squ nces $661-688$ and $1703-1709$ of PTEN ger (Fig. १) Dual luciferase reporter gene assay ig. b) showed that the relative luciferase activity of P. Vou- WT was decreased $(p<0.05)$, while there was no s. Mficant difference in luciferase activity of PTEN 1703-WT and PTEN-MUT co-transfected with miR29b-3p mimic $(p>0.05)$, suggesting that miR-29b-3p could specifically bind to the 661 site of PTEN gene. The level of PTEN in response to miR-29b-3p overexpression in cortical neurons and BMECs (Fig. 5c, d) showed that, upon miR-29b-3p overexpression, PTEN was significantly downregulated at both mRNA and protein levels in neurons and BMECs $(p<0.05)$. These data consistently showed that PTEN could be a target gene of miR-29b-3p.

Further, we probed into the effect of PTEN on ischemic brain injury. First, we found that the expression of PTEN was significantly upregulated in MCAO rats and OGD cells at both mRNA and protein levels with RTqPCR and Western blot analysis (Fig. 5e, f). OGD neurons or BMECs were infected with lentivirus harboring miR-29b-3p and/or PTEN shRNA. As shown in Fig. 5g, $h$, the expression of Bax and cleaved caspase- 3 was significantly decreased and Bcl-2 was upregulated in neurons after PTEN was downregulated, while the expression of angiogenesis-promoting proteins (VEGFA and VEGFR2) was significantly increased in BMECs, findings in line with the effects of miR-29b-3p overexpression. In addition, the silencing of PTEN could also enhance tube formation induced by miR-29b-3p overexpression in BMECs, and further inhibit pro-apoptosis gene expression (Fig. 5i). All of these results suggested that miR-29b-3p overexpression promoted tube formation of BMECs and repressed neuronal apoptosis by targeting PTEN. 


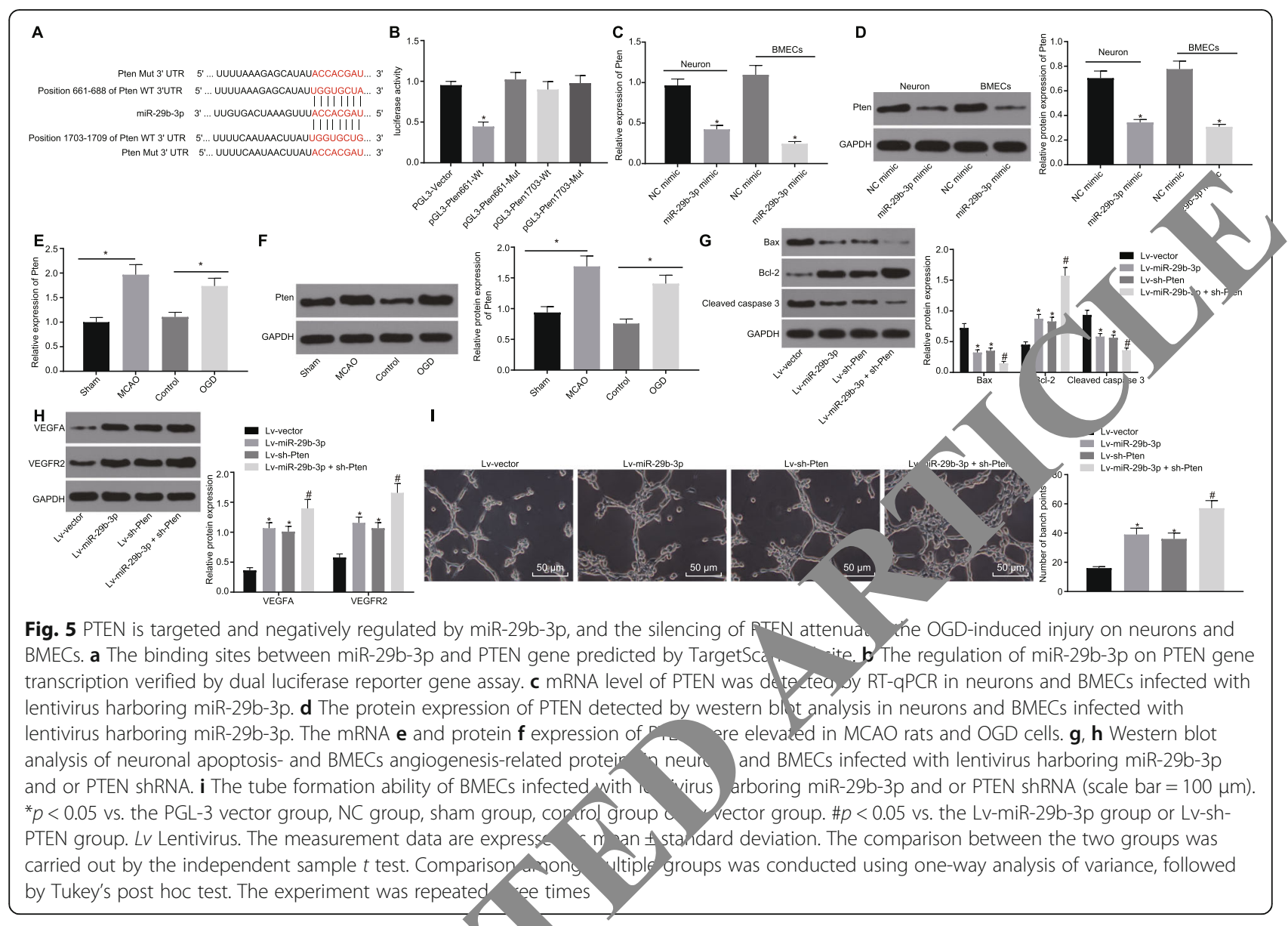

BMSC-derived exosomal miR-29b-3p a civates the A.t signaling pathway via targeting PTEN

As PTEN was reported to negatively late Akt signaling pathways [34], we tested w her Akt participated in the regulation of miR-29b-3p in a culture system of OGD cortical neurons a BMECs with BMSCs. The phosphorylation of $y$ olevated in OGD cortical neurons and BMF $\mathrm{Cs}$ co-cultured with miR-29b-3p expressed BMs (Fig. 6a b, e, f). Whereas, the phosphorylation of Ak as, suppressed by miR-29b-3p inhibitor, farther suppy,ting that miR-29b-3p blocked the activatio the Akt signaling pathway. Upon silencing of PMEN, Dhosphorylation of Akt was enhanced in th GD cortical neurons and BMECs (Fig. 6c, d, g, h). C ectrery, miR-29b-3p inhibits PTEN expression and furth promotes Akt activation.

\section{BMSC-derived exosomal miR-29b-3p alleviates the brain injury in MCAO rats}

Finally, we examined whether BMSC-derived exosomes play a beneficial role in the brain injury of MCAO rats. One hour after surgery, exosomes from untreated BMSCs (MSCs-Exo) or BMSCs expressing miR-29b-3p agomir (MSCs-Exo-miR-29b-3p agomir) were injected stereotactically into the brain. As shown in Fig. 7a, b, 2 weeks later, the volume of brain injury in the MSCsExo and MSCs-Exo-miR-29b-3p agomir groups was significantly less than that in the saline group, and the injury in the MSCs-Exo-miR-29b-3p agomir group was significantly less than that in the MSCs-Exo group. Besides, compared with the saline group, miR-29b-3p was significantly upregulated in the MSCs-Exo and MSCsExo-miR-29b-3p agomir groups, while the PTEN was significantly downregulated as detected by both RTqPCR and immunohistochemistry staining. Specifically, the alteration of miR-29b-3p and PTEN was more highly significant in the MSCs-Exo-miR-29b-3p agomir group (Fig. 7c-e). The apoptosis- and angiogenesis-related proteins (Fig. 7f, g) and TUNEL staining showed that the apoptotic level of the injured sites was attenuated in the MSCs-Exo and MSCs-Exo-miR-29b-3p agomir groups, which was more prominent in the MSCs-Exo-miR-29b$3 \mathrm{p}$ agomir group. In addition, the expression of $\mathrm{Bcl}-2$, VEGFA , and VEGFR2 was enhanced, while that of Bax and cleaved caspase-3 was reduced in the MSCs-Exo and MSCs-Exo-miR-29b-3p agomir groups; the difference was more pronounced in BMSC-exosome-miR29b-3p agomir group (Fig. 7h, i). In accordance, the 


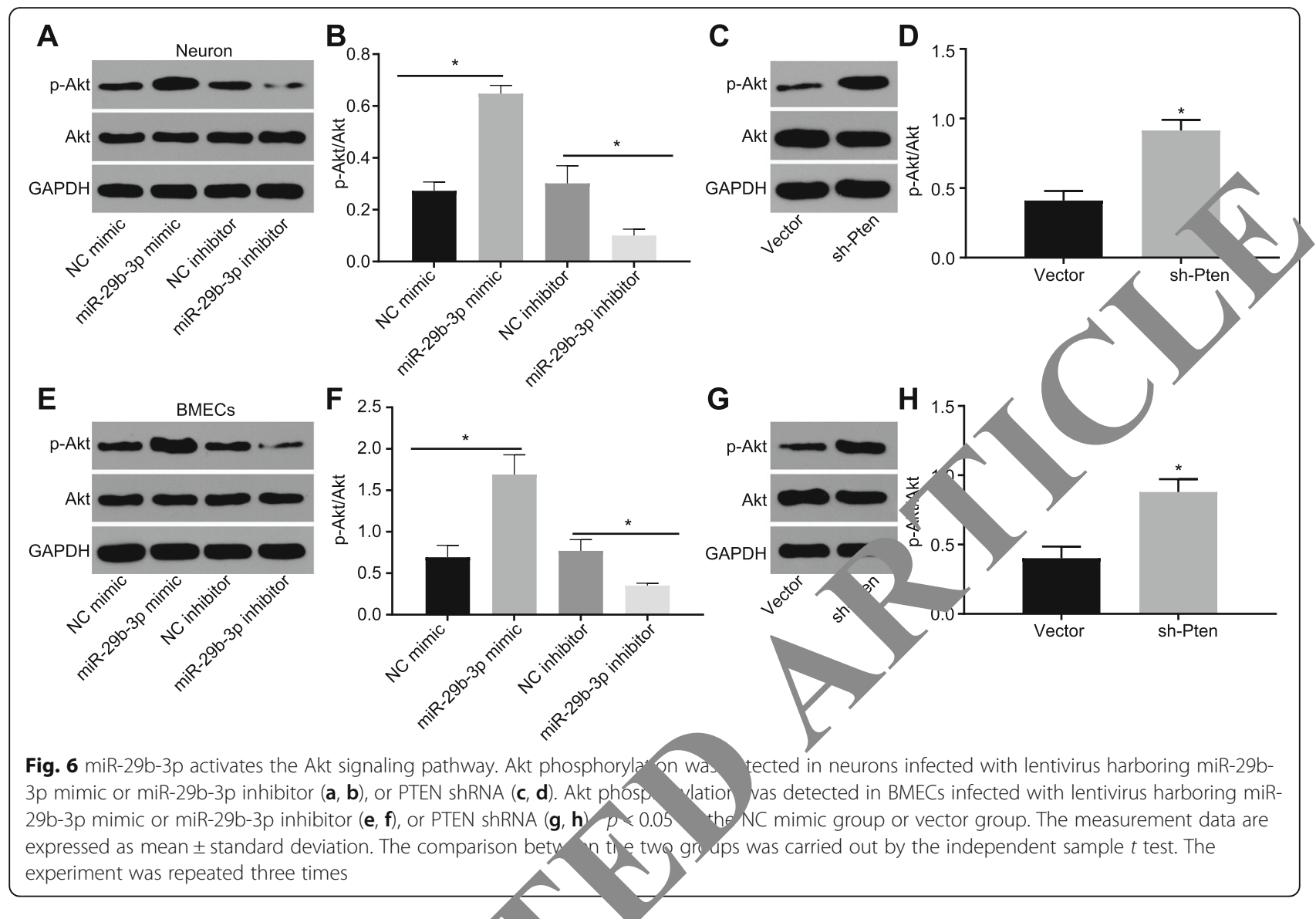

immunohistochemistry staining of CD 31 sho that angiogenesis was increased in the MS Cs-Exo and $1 / \mathrm{HCS}$ Exo-miR-29b-3p agomir groups, ar the argiogenesis promotion was significantly more no $\mathrm{I}_{\mathbf{v}}$ in the MSCsExo-miR-29b-3p agomir group $\Gamma_{i j}$. 7j, k). The results showed that BMSC-derived exo or could alleviate the brain injury in MCAO a and that miR-29b-3p agomir could further prom th honeticial effects of BMSCderived exosomes the rain injury.

\section{Discussion}

MSCs hose gained m, ch interest in the therapy for various disc and have also shown augmented protectio the at ment of neonatal hypoxic-ischemic brain iury 35]. R.ecently, exosomes derived from MSCs have bu tound to carry various kinds of mediators, miRNAs and steins, which can mediate the function of MSCs $[10,36,37]$. Besides, the reports of altered expression of miRNAs in hypoxic-ischemic brain injury suggest that miRNAs may participate in the pathogenesis of this model [38]. Based on this fact, we explored the role of miR-29b-3p in the BMSC-derived exosomes and in neuronal apoptosis and BMEC angiogenesis in the hypoxic-ischemic brain injury. Collectively, the data of the study revealed that BMSC-derived exosomal miR- 29b-3p could indeed suppress neuronal apoptosis and promote angiogenesis of BMECs through the downregulation of PTEN and activation of Akt signaling pathway.

First, we found that miR-29b-3p was significantly downregulated and PTEN was upregulated in MCAO rats, and OGD neurons and BMECs. The miR-29 family consists of miR-29a, miR-29b (b1 and b2 which are identical mature miRNAs), and miR-29c, with the mature miRNAs differing only in two or three bases. The expression of miR-29b-3p was found to be increased in osteoarthritis compared to the healthy neck-of-femur [39]. Indeed, miR-29b-3p potentiates chondrocyte apoptosis and facilitates the occurrence and progression of osteoarthritis [40]. Also, BMSC-specific overexpression of miR-29b-3p induced insulin resistance in young mice [41]. A previous study showed that miR-29b-3p protects against hypoxia-induced cell apoptosis [42]. PTEN is generally understood to be tumor-inhibiting protein but also plays important roles in neurological diseases [43]. For example, knockdown of PTEN was reportedly able to protect mouse brain from ischemic injury [44]. Second, we found that the apoptosis was increased in MCAO rats and OGD cells, and in BMSC-derived exosomes, whereas exosomal delivery of miR-29b-3p could inhibit the apoptosis in these models. The 


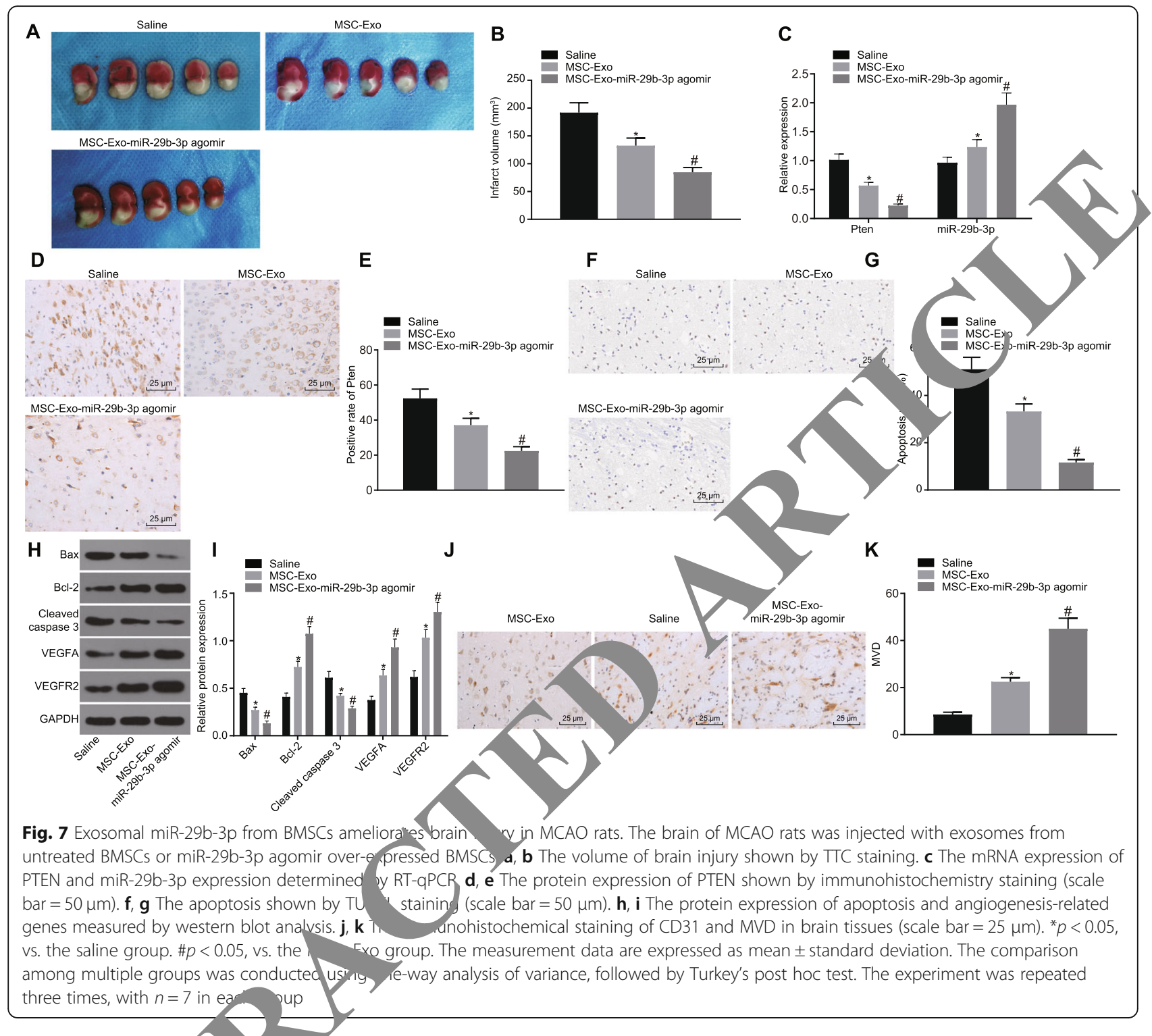

overexpression mi 9b-3p in OGD neurons or BMECs decrea $\%$ the exp1 ssion of Bax and cleaved caspase 3 and upregu $\mathrm{d}$ Bcl-2, confirming the inhibition of miR-2sb-3p on cel apoptosis.

Emers chta support a helpful role for angiogenesis and euro cvlar repair, together with a close intertior betwe n angiogenesis and neurogenesis [45]. Isch 1c suroke manifests as an ischemic core where neur death proceeds rapidly, which is surrounded by a vulnerable peri-infarct area, whereas angiogenic blood vessels near the infarct function as a scaffold for neuronal progenitors, empowering neurons to migrate toward the peri-ischemic regions [46]. Neurogenesis and angiogenesis could thus be viewed as a complex and dynamic coupling with complex cross-talk between neurons and endothelial cells through vascular endothelial growth factor (VEGF), neurotrophins, as well as their cognate receptors on both neurons and endothelial cells [47]. Specifically, the 15-LO-1/15-HETE system presents a potential approach for aiding neurobehavioral recovery after cerebral ischemic stroke, a beneficial effect mediated by upregulation of VEGF and subsequent promotion of angiogenesis in the ischemic brains [48]. Exosomal signaling during hypoxia regulates angiogenesis and migration of MECs [49], and recent research shows that hypoxia stimulates the release of exosomes in various tumor types, culminating in the activation of vascular cells and angiogenesis [50]. According to a recent review, cell-based and cell free (exosomes, extracellular vesicles, microRNAs) therapies have already been applied successfully for angiogenesis-mediated tissue regeneration and have great potential for treating ischemic heart disease, brain stroke, as well as bone defects [51]. Furthermore, exosome-derived communication between 
endothelial cells and cardiomyocytes shows the potential for induction of local neo-vascularization during cardiac injury, and furthermore, cardiomyocyte-derived exosomes carry a broad repertoire of miRNAs and proteins under conditions of glucose deprivation [52]. In another example, exosomes derived from miR-126-modified MSCs facilitated the angiogenesis and migration of human umbilical venous endothelial cells after spinal cord injury [53]. Besides, upregulation of miR-29b plays a promotive role in ischemiainduced angiogenesis [54], indicating that miR-29b-3p is a promising factor in the treatment of hypoxic-ischemic brain injury. In this study, we revealed that declining cerebral levels of miR-29b-3p in MCAO rat brain was related to decreased angiogenesis, as indicated by the deregulation of VEGFA, VEGFR2 and CD31. In addition, the injection of BMSCs-derived exosomes containing miR-29b-3p mimic could ameliorate the suppression of angiogenesis in MCAO rats, further supporting miR-29b-3p to be a potential factor for the treatment of hypoxia-ischemia brain injury.

Finally, we found that negative regulation of PTEN and activation of Akt mediated the effects of miR-29b-3p on the amelioration of brain injury caused by hypoxicischemia in the present models. The suppression of PTEN could activate the Akt signaling pathway and also mediated protection against ischemic neuronal death [43]. Besides, the inhibition of PTEN and activation of Akt by baicalen can suppress oxidant stress and scavenge free radicals in the ischemia/reperfusion injury model [44]. Finally, we note that the PI3K/Akt signaling pathway ${ }^{\circ} \mathrm{n}$ accelerate angiogenesis after ischemic stroke [5]. Our present report further confirmed the critical role TEN and Akt pathway in the pathogenesis and recove of hypoxic-ischemic brain injury.

\section{Conclusion}

These findings provided new ightwo the pathogenesis of hypoxic-ischemic brain $\mathrm{ln} v \mathrm{v}$, showing that miR29b-3p was downreg la a in MQAO rats and neurons under exposure to OGD. L ides, miR-29b-3p delivered in exosomes from MSCs accelerated angiogenesis of BMECs and $h \mathrm{~h}$ aronal apoptosis after ischemic stroke via targetir. DTEN and activating the Akt signaling pathr (Fig. \%). Thus, present results show that miR-29b-3 Nay well present a potential therapeutic target in the experimental treatment of hypoxic-ischemic bra injury.

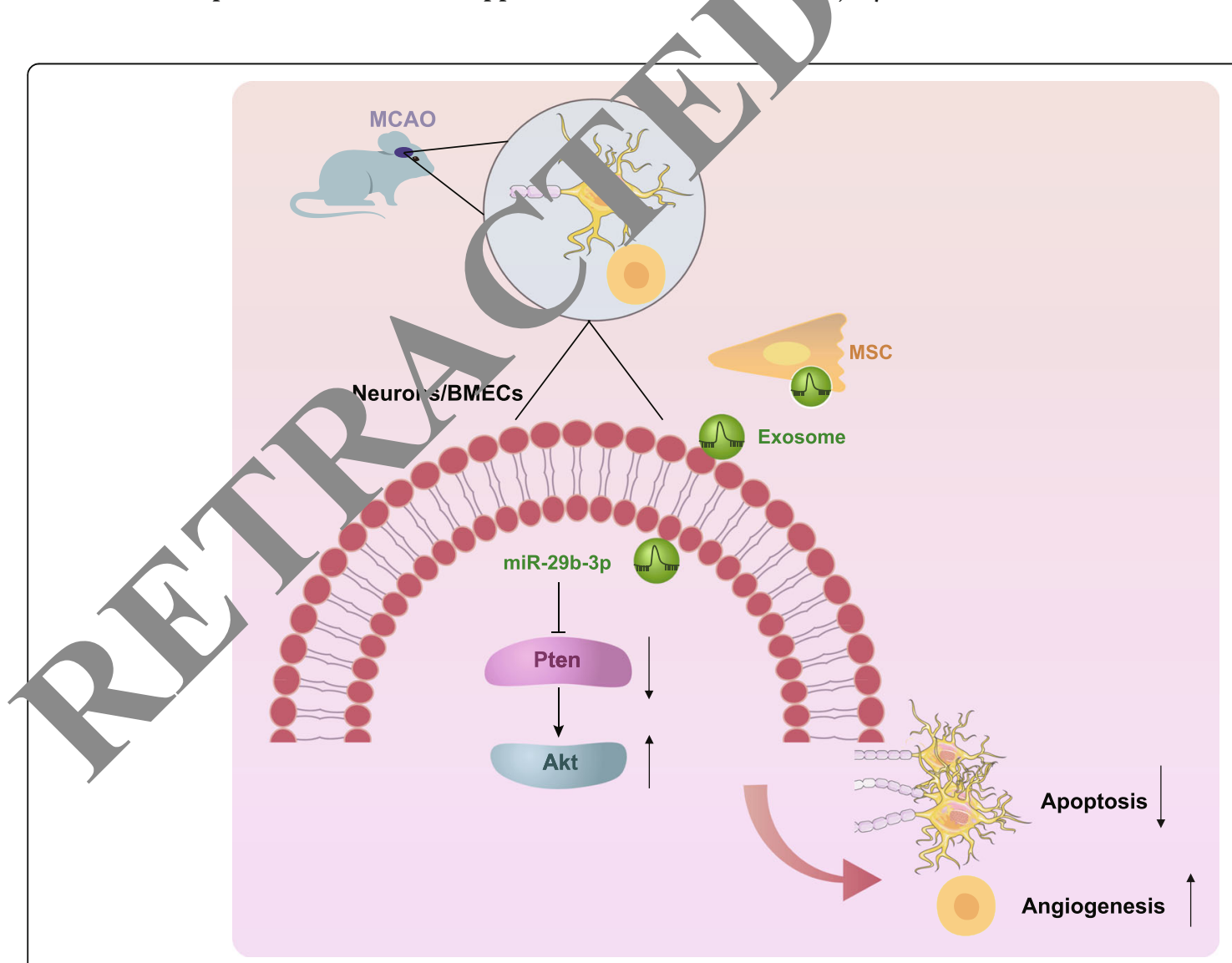

Fig. 8 BMSC-derived exosomal miR-29b-3p suppresses neuronal apoptosis and promotes BMECs angiogenesis through the downregulation of PTEN and activation of the Akt signaling pathway 


\section{Affiliated results Immunofluorescence assay}

After stable growth, cells were fixed with $4 \%$ paraformaldehyde at ambient temperature for $30 \mathrm{~min}$. After permeabilization and sealing, cells were incubated with primary mouse antibodies against CD90 (ab225, 1:1000), CD105 (ab11414, 1:1000), Vimentin (ab8978, 1:1000), Ecadherin (ab1416, 1:1000), CD31 (ab24590, 1:1000), and rabbit antibodies against CD44 (ab157107, 1:1000), NeuN (ab177487, 1:300), and MAP2 (ab32454, 1:1000) overnight at $4{ }^{\circ} \mathrm{C}$. Subsequently, the cells were incubated with fluorescent secondary antibody goat anti-mouse (ab6785, 1:1000) (green) or goat anti-rabbit (ab150075, 1: 000) (red) at ambient temperature for $60 \mathrm{~min}$ in the dark. All of the above antibodies were purchased from Abcam (Cambridge, UK). The cells were stained with DAPI for $10 \mathrm{~min}$ at ambient temperature and sealed with a sealing agent (36,308 ES11, Yeasen Biotechnology Co., Ltd., Shanghai, China), and finally observed under a fluorescence microscope (BX53, Olympus, Tokyo, Japan).

\section{Primary BMSCs, cortical neurons, and BMECs were successfully isolated}

BMSCs at passage 4 presented fibroblasts-like appearance as shown in Additional file 1: Figure S1A. Immanofluorescence assay displayed that BMSCs were $P_{4}$ for CD90, CD44, CD105, and negative for e-cadho and Vimentin (Additional file 1: Figure S1\%), ogestin. successful isolation of BMSCs.

The cultured cortical neurons adhe ing to the wall $3 \mathrm{~h}$ after seeding were mostly round d sphere-shaped, with a few protrusions. On the sixth ne neurons formed a complex neuroid (1) rional file 1: Figure $\mathrm{S} 1 \mathrm{C})$. The expression of neuron marn, nuclear proteins NeuN and MAP2 was ael ted by cellular immunofluorescence. The results neurons were positive for NeuN (Addit onal fiı Figure S1D), suggesting successful isolation cortical neurons.

BMECs a the thi lay presented a typical flagstone-like structure and a "vortex-like distribution" (Additional file 1: Figure S, The CD31 immunofluorescence assay confir. the ssful isolation of BMECs (Additional file 1:

\section{Supp.ementary information}

Supplementary information accompanies this paper at https://doi.org/10. 1186/s12974-020-1725-8.

Additional file 1. Figure S1. Isolation and identification of primary BMSCs, cortical neurons and BMECs. Light microscopic observation ( $A$; scale bar $=50 \mu \mathrm{m})$ and immunofluorescence staining (B; scale bar = $25 \mu \mathrm{m})$ of CD90, CD44, CD105, E-cadherin and Vimentin in primary BMSCs. Light microscopic observation (C; scale bar $=50 \mu \mathrm{m})$ and immunofluorescence staining (D; scale bar $=50 \mu \mathrm{m})$ of NeuN and MAP2 in

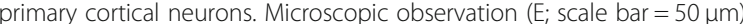
and CD31 immunofluorescence staining ( $F$; scale bar $=25 \mu \mathrm{m}$ ) of BMECs. (EPS $27896 \mathrm{~kb})$

\section{Acknowledgments}

We would like to give our sincere appreciation to the colleagues for their helpful comments on this article.

\section{Authors' contributions}

Conception and design: KH, JZ, YZ, KX; analysis and interpretation: GL, BX, collection: $K H, J Z, Y Z, K X$; writing the article: GL, BX, JY; critical vision of the ar $\mathrm{KH}, J Z, Y Z, K X$; final approval of the article: GL, BX, JY; statisticas vvsis: KH,.$\} Y Z, K X$; obtained funding: GL, BX, JY. All authors read and apperver the mar ascript.

Funding

None.

Availability of data and materia?

The datasets generated/analyze uring the ont study are available.

Ethics approval and concent to par inate

The present study wa ap ved by the Ethics Committee of The First Hospital of Jilin Un ity. Lnrocedures involving animals were conducted in line with the regula sor trunstitutional animal care and use committee (ethics numb $028-415$ ).

Consent for publ

Consent for pulication was obtained from the participants. Cor. ing interests

The au rs declare that they have no competing interests.

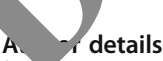

1De. partment of Neurosurgery, The First Hospital of Jilin University, No. 1 inmin Avenue, Changchun 130021, Jilin, People's Republic of China. ${ }^{2}$ Department of Neurology, The First Hospital of Jilin University, Changchun 130021, People's Republic of China.

Received: 23 July 2019 Accepted: 24 January 2020

Published online: 03 February 2020

\section{References}

1. Saeed E, Ali R, Jalal-ud-din M, Saeed A, Jadoon RJ, Moiz M. Hypercholesterolemia in patients of ischemic stroke. J Ayub Med Coll Abbottabad. 2015;27:637-9.

2. $\quad$ Song H, Zhou H, Qu Z, Hou J, Chen W, Cai W, Cheng Q, Chuang DY, Chen S, Li S, Li J, Cheng J, Greenlief CM, Lu Y, Simonyi A, Sun GY, Wu C, Cui J, Gu Z. From Analysis of Ischemic Mouse Brain Proteome to Identification of Human Serum Clusterin as a Potential Biomarker for Severity of Acute Ischemic Stroke. Transl Stroke Res. 2019;10(5):546-56.

3. Sommer CJ. Ischemic stroke: experimental models and reality. Acta Neuropathol. 2017:133:245-61.

4. Wang Y, Luo J, Li SY. Nano-Curcumin simultaneously protects the bloodbrain barrier and reduces M1 microglial activation during cerebral ischemiareperfusion injury. ACS Appl Mater Interfaces. 2019;11:3763-70.

5. Zerna C, Thomalla G, Campbell BCV, Rha JH, Hill MD. Current practice and future directions in the diagnosis and acute treatment of ischaemic stroke. Lancet. 2018;392:1247-56.

6. Won SJ, Kim DY, Gwag BJ. Cellular and molecular pathways of ischemic neuronal death. J Biochem Mol Biol. 2002;35:67-86.

7. Ruan L, Wang B, ZhuGe Q, Jin K. Coupling of neurogenesis and angiogenesis after ischemic stroke. Brain Res. 1623;2015:166-73.

8. Lee JA, Kim BI, Jo CH, Choi CW, Kim EK, Kim HS, Yoon KS, Choi JH. Mesenchymal stem-cell transplantation for hypoxic-ischemic brain injury in neonatal rat model. Pediatr Res. 2010;67:42-6.

9. Ward MR, Abadeh A, Connelly KA. Concise review: rational use of mesenchymal stem cells in the treatment of ischemic heart disease. Stem Cells Transl Med. 2018;7:543-50.

10. Zhang D, Lee H, Zhu Z, Minhas JK, Jin Y. Enrichment of selective miRNAs in exosomes and delivery of exosomal miRNAs in vitro and in vivo. Am J Physiol Lung Cell Mol Physiol. 2017;312:L110-L21. 
11. Zhu CG, Liu YX, Wang H, Wang BP, Qu HQ, Wang BL, Zhu M. Active form of vitamin $\mathrm{D}$ ameliorates non-alcoholic fatty liver disease by alleviating oxidative stress in a high-fat diet rat model. Endocr J. 2017;64:663-73.

12. Birch D, Britt BC, Dukes SC, Kessler JA, Dizon ML. MicroRNAs participate in the murine oligodendroglial response to perinatal hypoxia-ischemia. Pediatr Res. 2014;76:334-40.

13. Majdi A, Mahmoudi J, Sadigh-Eteghad S, Farhoudi M, Shotorbani SS. The interplay of microRNAs and post-ischemic glutamate excitotoxicity: an emergent research field in stroke medicine. Neurol Sci. 2016;37:1765-71.

14. Widlansky ME, Jensen DM, Wang J, Liu Y, Geurts AM, Kriegel AJ, Liu P, Ying R, Zhang G, Casati M, Chu C, Malik M, Branum A, Tanner MJ, Tyagi S, Usa K, Liang M. miR-29 contributes to normal endothelial function and can restore it in cardiometabolic disorders. EMBO Mol Med. 2018;10(3).

15. Dai Y, Mao Z, Han X, Xu Y, Xu L, Yin L, Qi Y, Peng J. MicroRNA-29b-3p reduces intestinal ischaemia/reperfusion injury via targeting of TNF receptor-associated factor 3. Br J Pharmacol. 2019;176:3264-78.

16. Garcia-Junco-Clemente P, Golshani P. PTEN: a master regulator of neuronal structure, function, and plasticity. Commun Integr Biol. 2014;7:e28358.

17. Qu L, Gao Y, Sun H, Wang H, Liu X, Sun D. Role of PTEN-Akt-CREB signaling pathway in nervous system impairment of rats with chronic Arsenite exposure. Biol Trace Elem Res. 2016;170:366-72.

18. Hung PL, Hsu MH, Yu HR, Wu KLH, Wang FS. Thyroxin Protects White Matter from Hypoxic-Ischemic Insult in the Immature Spraque-Dawley Rat Brain by Regulating Periventricular White Matter and Cortex BDNF and CREB Pathways. Int J Mol Sci. 2018;19(9).

19. Huang W, Shao M, Liu H, Chen J, Hu J, Zhu L, Liu F, Wang D, Zou Y, Xiong $Y$, Wang X. Fibroblast growth factor 21 enhances angiogenesis and wound healing of human brain microvascular endothelial cells by activating PPARgamma. J Pharmacol Sci. 2019;140:120-7.

20. Longa EZ, Weinstein PR, Carlson S, Cummins R. Reversible middle cerebral artery occlusion without craniectomy in rats. Stroke. 1989;20:84-91.

21. Zhang G, Ge M, Han Z, Wang S, Yin J, Peng L, Xu F, Zhang Q, Dai Z, Xie L, Y, Si J, Ma K. Wnt/beta-catenin signaling pathway contributes to isoflurane postconditioning against cerebral ischemia-reperfusion injury and is possibly related to the transforming growth factorbeta1/Smad3 s/ pathway. Biomed Pharmacother. 2019;110:420-30.

22. Jiang $M$, Wang $H$, Jin $M$, Yang $X$, Ji H, Jiang $Y$, Zhang $H$, Wu $\ulcorner W U G$, L et al. Exosomes from MiR-30d-5p-ADSCs reverse acute is ic strokeinduced, autophagy-mediated brain injury by promoting $\mathrm{M} 2 \mathrm{~h}$. alial/ macrophage polarization. Cell Physiol Biochem.

23. Bederson JB, Pitts LH, Tsuji M, Nishimura MC, Da middle cerebral artery occlusion: evaluation of $\mathrm{t}$ model and development of a neurologic examination. Stroke. 1986:17:472

24. Huang $X$, Ding J, Li Y, Liu W, Ji J, Wang -4 . Wang $X$ PEDF modified adipose-derived mesend ischemia-reperfusion injury by regulation of 7 atv, agy and apoptosis. Exp Cell Res. 2018:371:269-77.

25. Yavin Z, Yavin E. Survival ad ma ration of Cerebral neurons on poly $(\mathrm{L}-$ lysine) surfaces in the arse Jev Biol. 1980;75:454-9.

26. Qin AP, Liu CF, Qin Y Y, Fong Yu M, Yang L, Liu J, Qin ZH, Zhang HL. Autophagy was a ted in injur. astrocytes and mildly decreased cell survival follorng gh and oxygen deprivation and focal cerebral ischemia. tophagy. 2 .738-53.

27. Goldb g MP, Choi DW. Lombined oxygen and glucose deprivation in cortic II ture. calcium-dependent and calcium-independent mechanı of ne ronal injury. J Neurosci. 1993;13:3510-24. g L, Pe an L, Guo B, Li Y, Duan R, Yao Y, Xue B, Chen X, Jia Y. $M \in$ unchvmal stem cell-derived exosomes reduce $A 1$ astrocytes via uation of phosphorylated NFkappaB P65 subunit in spinal cord Cell Physiol Biochem. 2018:50:1535-59.

9. Ay, SM, Abrahamse H, Houreld NN. The role of photobiomodulation on gene expression of cell adhesion molecules in diabetic wounded fibroblasts in vitro. J Photochem Photobiol B. 2016;161:368-74.

30. Weidner N. Tumor angiogenesis: review of current applications in tumor prognostication. Semin Diagn Pathol. 1993;10:302-13.

31. Dai Y, Mao Z, Han X, Xu Y, Xu L, Yin L, Qi Y, Peng J. MicroRNA-29b-3p reduces intestinal ischemia/reperfusion injury via targeting of TRAF3. $\mathrm{Br} J$ Pharmacol. 2019;176(17):3264-78

32. Cosenza S, Ruiz M, Toupet K, Jorgensen C, Noel D. Mesenchymal stem cells derived exosomes and microparticles protect cartilage and bone from degradation in osteoarthritis. Sci Rep. 2017;7:16214.
33. Thomi G, Surbek D, Haesler V, Joerger-Messerli M, Schoeberlein A. Exosomes derived from umbilical cord mesenchymal stem cells reduce microglia-mediated neuroinflammation in perinatal brain injury. Stem Cell Res Ther. 2019;10:105.

34. Carnero A, Blanco-Aparicio C, Renner O, Link W, Leal JF. The PTEN/PI3K/AKT signalling pathway in cancer, therapeutic implications. Curr Cancer Drug Targets. 2008;8:187-98.

35. Herz J, Koster C, Reinboth BS, Dzietko M, Hansen W, Sabir H, van Velthoven C, Bendix I, Felderhoff-Muser U. Interaction between hypothermia and delayed mesenchymal stem cell therapy in neonatal hypoxic-is emil brain injury. Brain Behav Immun. 2018;70:118-30.

36. Bai L, Shao H, Wang H, Zhang Z, Su C, Dong L, Yu B, Chen X, Li X, . gg X Effects of Mesenchymal stem cell-derived exosomes hexperiment autoimmune uveitis. Sci Rep. 2017;7:4323.

37. Shamili FH, Bayegi HR, Salmasi Z, Sadri K, M Ramezani M, Abnous K. Exosomes derived fi mesenchymal stem cells with effective ant melanoma model. Int J Pharm. 2018;549:21

38. Cui $H$, Yang L. Analysis of microRNA exp on de microarray of the cerebral cortex after hypoxic-ischen brain v.J Craniofac Surg. 2013;24:2147-52

39. Le LT, Swingler TE, Crowe N ncent TL, Ba MJ, Donell ST, Delany AM, Dalmay T, Young DA, Cla, $\mathbb{N}$. microRINA-29 family in cartilage homeostasis and osteoart ritis. . Med (Berl). 2016;94:583-96.

40. Chen L, Li Q, Wang Zheng H, L. S, He F, Zhang H, Ma S, Mei J, Yu J. MiR29b-3p promotec hondi yte apoptosis and facilitates the occurrence and development of O. a argeting PGRN. J Cell Mol Med. 2017;21:3347-59.

41. Su T, Xiao Y, Xiao Y, Q, Li C, Huang Y, Deng Q, Wen J, Zhou F, Luo XH. Bone $m$ wiw Mesench, al stem cells-derived exosomal MiR-29b-3p regulates ay nciated insulin resistance. ACS Nano. 2019;13:2450-62.

42. Zhou S, Le D, Bu, , Han H, Zhao S, Wang Y. MicroRNA-29b-3p targets SPARC gen to protect cardiocytes against autophagy and apoptosis in

rpoxic-indy, ced H9c2 cells. J Cardiovasc Transl Res. 2019:12:358-65.

43. $\mathrm{gg} \mathrm{N}$, El-Hayek YH, Gomez E, Wan Q. Phosphatase PTEN in neuronal and brain disorders. Trends Neurosci. 2007;30:581-6.

, Wu J, Xu K, Cai F, Gu J, Ma L, Chen J. Neuroprotection by baicalein in chemic brain injury involves PTEN/AKT pathway. J Neurochem. 2010;112:1500-12. Arai K, Jin G, Navaratna D, Lo EH. Brain angiogenesis in developmental and pathological processes: neurovascular injury and angiogenic recovery after stroke. FEBS J. 2009;276:4644-52.

46. Dzietko M, Derugin N, Wendland MF, Vexler ZS, Ferriero DM. Delayed VEGF treatment enhances angiogenesis and recovery after neonatal focal rodent stroke. Transl Stroke Res. 2013;4:189-200.

47. Madri JA. Modeling the neurovascular niche: implications for recovery from CNS injury. J Physiol Pharmacol. 2009;60(Suppl 4):95-104.

48. Chen L, Zhu YM, Li YN, Li PY, Wang D, Liu Y, Qu YY, Zhu DL, Zhu YL. The 15-LO-1/15-HETE system promotes angiogenesis by upregulating VEGF in ischemic brains. Neurol Res. 2017;39:795-802.

49. Salomon C, Ryan J, Sobrevia L, Kobayashi M, Ashman K, Mitchell M, Rice GE. Exosomal signaling during hypoxia mediates microvascular endothelial cell migration and vasculogenesis. PLoS One. 2013;8:e68451.

50. Sahebi R, Langari H, Fathinezhad Z, Bahari Sani Z, Avan A, Ghayour Mobarhan M, Rezayi M. Exosomes: new insights into cancer mechanisms. J Cell Biochem. 2020;121:7-16.

51. Reddy LVK, Murugan D, Mullick M, Begum M ET, Sen D. Recent Approaches for Angiogenesis in Search of Successful Tissue Engineering and Regeneration. Curr Stem Cell Res Ther. 2019. https://doi.org/10.2174/ 1574888X14666191104151928. [Epub ahead of print].

52. Garcia NA, Ontoria-Oviedo I, Gonzalez-King H, Diez-Juan A, Sepulveda P. Glucose starvation in cardiomyocytes enhances exosome secretion and promotes angiogenesis in endothelial cells. PLoS One. 2015;10:e0138849.

53. Huang $J H, X u Y$, Yin XM, Lin FY. Exosomes Derived from miR-126-modified MSCs Promote Angiogenesis and Neurogenesis and Attenuate Apoptosis after Spinal Cord Injury in Rats. Neuroscience. 2020;424:133-45.

54. Zhu ML, Yin YL, Ping S, Yu HY, Wan GR, Jian X, Li P. Berberine promotes ischemia-induced angiogenesis in mice heart via upregulation of microRNA29b. Clin Exp Hypertens. 2017;39:672-9.

55. Yu X, Peng Y, Liang H, Fu K, Zhao Z, Xie C, Zhou L, Zhang K. TSLP/TSLPR promote angiogenesis following ischemic stroke via activation of the PI3K AKT pathway. Mol Med Rep. 2018;17:3411-7.

\section{Publisher's Note}

Springer Nature remains neutral with regard to jurisdictional claims in published maps and institutional affiliations. 